\title{
Lessons learned from HIV-1 vaccine trials: new priorities and directions
}

\author{
Andrew J McMichael \& Barton F Haynes \\ A vaccine against human immunodeficiency virus (HIV) seems to be on the horizon. Correlates of risk of infection for \\ the RV144 trial have been found. There is understanding of what makes HIV envelope-specific antibodies broadly \\ neutralizing and new $T$ cell vaccine approaches can overcome virus variability.
}

\begin{abstract}
$\Delta$ s recently as 3 years ago, research into Avaccines against human immunodeficiency virus (HIV) was stuck in the doldrums. Vaccines based on the virus envelope protein gp120 (AIDSVAX B/E gp120) had failed to demonstrate protection against infection with HIV in clinical trials ${ }^{1,2}$. Delineation of the envelope antigenic structure with monoclonal antibodies identified only rare sites of weakness on the virus and even rarer antibodies. High hopes for a vaccine directed against the group antigen (Gag), polymerase ( $\mathrm{Pol}$ ) and negative factor (Nef) proteins of HIV with adenovirus type 5 as the vector, aimed at stimulating strong $\mathrm{CD}^{+} \mathrm{T}$ cell responses, had been dashed, with no protection noted and safety concerns raised about the vector ${ }^{3}$.
\end{abstract}

Then a surprising and completely unexpected result arrived. The approach of combining the AIDSVAX B/E gp120 vaccine with the $\mathrm{CD}^{+} \mathrm{T}$ cell-stimulating ALVAC canarypox vaccine (ALVAC-HIV/AIDSVAX B/E) in the RV144 trial in Thailand, tested in the face of considerable criticism, showed an estimated efficacy of $31.2 \%$ for protection against the acquisition of HIV type 1 (HIV-1 $)^{4,5}$. This finding has reinvigorated the field, leading many to believe that development of a vaccine really is possible (Fig. 1).

Andrew J. McMichael is with the Weatherall Institute of Molecular Medicine, John Radcliffe Hospital, University of Oxford, Oxford, UK. Barton F. Haynes is with the Duke Human Vaccine Institute and the Center for HIV/AIDS Vaccine Immunology, Department of Medicine, Duke University School of Medicine, Durham, North Carolina, USA. e-mail: andrew.mcmichael@ndm.ox.ac.uk

\section{The RV144 vaccine trial}

The RV144 trial is not without its critics ${ }^{6}$. The investigators themselves have been among the most cautious, avoiding hyperbole. Gilbert et al. have provided a robust statistical critique of the results ${ }^{7}$. They strongly support the primary analysis of the 'modified intention-totreat' group, which removes volunteers who became infected between screening and first vaccination. This analysis gave a $P$ value of 0.04 ; that is, a $4 \%$ chance of a false-positive efficacy result ${ }^{7}$. In a post-hoc analysis with Bayesian statistics, which was not prespecified in the study's statistical-analysis plan, they concluded that the chance of no vaccine efficacy is $\leq 22 \%$, which would leave a good chance that the vaccine indeed worked ${ }^{6}$. Because the RV144 trial of ALVAC-HIV/ AIDSVAX $B / E$ is the first trial of a vaccine against HIV-1 to show any degree of efficacy, it provides the first opportunity to plot a way forward for a globally effective vaccine grounded in clinical research.

Further study of the RV144 trial affords an important opportunity for the field to define correlates of protection in humans that will aid the design of more-effective vaccines in the future. The vaccine assessed in this trial did not stimulate broadly neutralizing antibodies (BnAbs) able to neutralize a broad range of transmitted or founder virus isolates $^{8}$, generally regarded as the sine qua non of an HIV-1 vaccine, nor did it stimulate measurable responses by $\mathrm{CD} 8^{+}$cytolytic $\mathrm{T}$ cells $^{4,5}$. Instead, the ALVAC-HIV/AIDSVAX $\mathrm{B} / \mathrm{E}$ vaccine induced $\mathrm{CD} 4^{+} \mathrm{T}$ cell and antibody-dependent cell-mediated cytotoxicity responses and induced only neutralizing antibodies to the easy-to-neutralize (tier 1)
HIV-1 strains ${ }^{8}$. Haynes, Kim and colleagues ${ }^{9}$ coordinated a detailed attack on this problem by comparing a range of immune parameters in 41 vaccinated participants who became infected and 205 vaccinated subjects who did not become infected. Using robust assays, they found two strong correlates with infection risk. One was the plasma concentration of immunoglobulin $\mathrm{G}$ (IgG) antibody specific for the V1V2 loop region of envelope gp120, which was inversely correlated with infection risk. The other was high plasma concentrations of IgA antibody to HIV-1 Env, which were directly correlated with acquisition of infection. These findings have generated the following two hypotheses: that high concentrations of plasma antibodies specific for V1V2 are involved in protection against acquisition of HIV-1; and that high plasma concentrations of IgA to Env mitigate the effects of protective antibodies ${ }^{9}$.

Many laboratories are now working to determine if those two correlates of risk in the RV144 trial are related mechanistically to the degree of protection noted in the trial or whether they are only surrogate markers for other factors. For example, if the types of V1V2-specific antibodies induced by the ALVAC-HIV/AIDSVAX B/E vaccine can be shown, after passive infusion into rhesus macaques, to protect against challenge with chimeric SHIV (simian immunodeficiency virus (SIV) with an HIV-1 envelope), then vaccines could be designed to induce V1V2specific antibodies at concentrations higher than those seen in the RV144 trial.

There is one additional caveat about the results noted above. When the AIDSVAX B/E vaccine was used alone in the VAX003 trial 


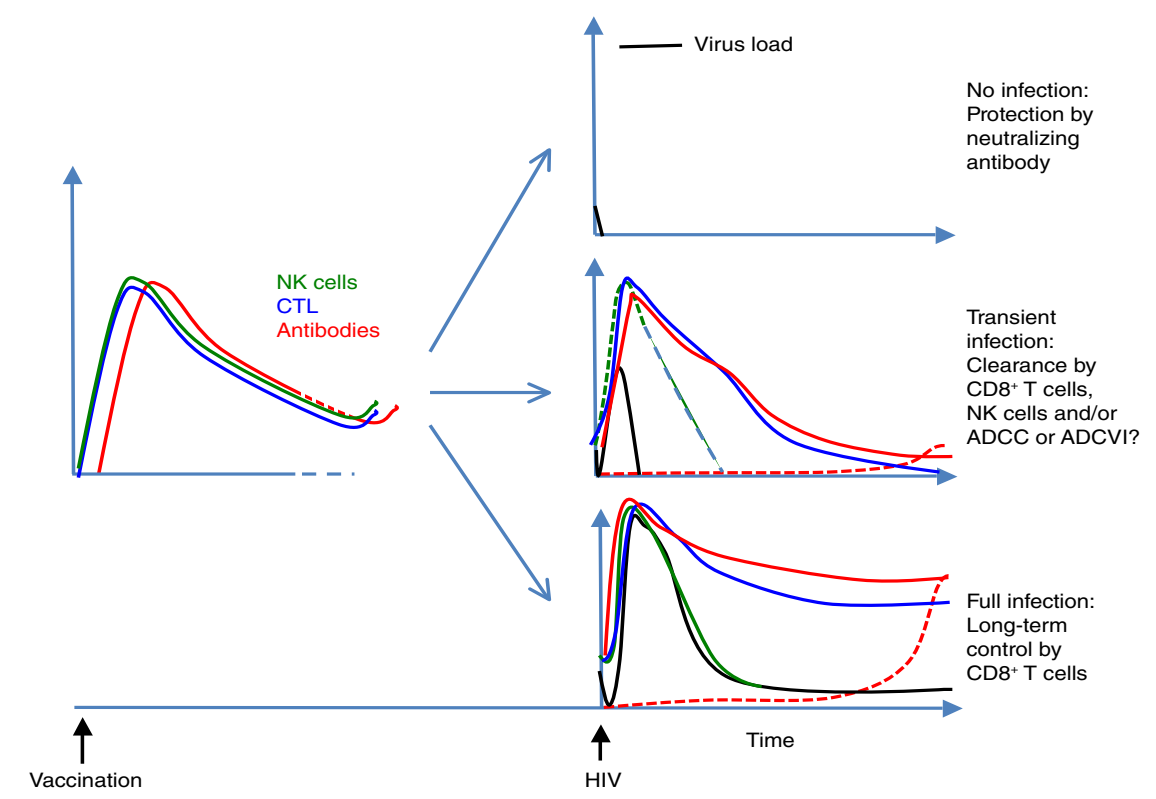

Figure 1 Three possible protective outcomes of an HIV-1 vaccine. The immune response to a vaccine (left) and possible outcomes after later exposure to HIV-1 (right). Top right, the antibody response after vaccination is strong and broad enough to neutralize the HIV-1 before infection can be established. Middle right, the immune responses, $\mathrm{CD}^{+} \mathrm{T}$ cells, non-neutralizing antibodies and/or natural killer cells (NK cells) that mediate antibody-dependent cell-mediated cytotoxicity (ADCC) clear the infection within days of exposure. Bottom right, the infection is established, but the CD8 ${ }^{+} \mathrm{T}$ cell response, possibly aided by antibody and innate responses, establishes very good control of the virus with a very low virus load and prolonged survival (without the need for antiretroviral therapy). CTL, cytotoxic T lymphocyte; ADCVI, antibody-dependent cell-mediated viral inhibition.

in Thailand of subjects who were high-risk intravenous drug users, it showed no effi$\mathrm{cacy}^{2}$. In contrast, the Thai volunteer cohort in the RV144 trial was mainly heterosexual with a relatively low risk of infection with HIV-1. In the RV144 trial, the acquisitionof-infection rate was only $0.19 \%$ per year, in contrast to the rate of $\sim 5 \%$ per year for some high-risk cohorts ${ }^{10}$. Sexual acquisition of HIV-1 infection is most commonly the result of a single virus despite exposure to thousands of different virions per sexual $\operatorname{act}^{11}$. In other words, transmission is a rare event. Perhaps the ALVAC-HIV/AIDSVAX $\mathrm{B} / \mathrm{E}$ vaccine simply decreased the chance of acquisition marginally, increasing the average number of exposures needed for infection and, in doing so, delayed infection. In this setting, a small vaccine-efficacy effect would show up in a low-risk cohort monitored very closely over time, but it might not show up in a high-risk sexual-transmission cohort or in high-risk intravenous drug users.

Given the correlates study of the RV144 trial, it is worth noting that post-hoc analysis of the vaccine-efficacy study of the VAX004 AIDSVAX B/B gp120 vaccine, which failed to protect Thai intravenous drug users against HIV infection, suggests that vaccineinduced neutralizing antibody, CD4-blocking antibody and antibody-dependent cellular viral inhibition were associated with lower rates of infection in vaccinees ${ }^{1,12}$.

Several attempts are now in progress to extend the results of the RV144 study with vaccine constructs that will stimulate stronger Env-specific immune responses. The efficacy trials will take another 5 years, but if the results of the RV144 trial can be confirmed and improved on, this empirical approach could lead to a usable vaccine in the foreseeable future. However, it must also be recognized that the results of the RV144 trial might not be generalizable to other risk groups, and therefore it is essential that other strategies be pursued vigorously in parallel with efforts to improve on the prime-boost approach used with the ALVAC-HIV/AIDSVAX B/E vaccine.

\section{Broadly neutralizing antibodies}

Fortunately, there have also been exciting advances in the understanding of both BnAbs specific for HIV-1 (refs. 13,14) and $\mathrm{T}$ cell immunity ${ }^{15}$. For many years, the HIV antibody field was restricted to the study of a few monoclonal antibodies that identify the following three epitope sites on the envelope: the CD4-binding site, the membraneproximal external regions, and a particular pattern of glycans ${ }^{13,14}$. These antibodies are very rare, out of thousands made that are less active, and most of the protective antibodies have unusual features, such as a long complementarity-determining region 3 (CDR3) in the heavy-chain variable $\left(\mathrm{V}_{\mathrm{H}}\right)$ region, a large number of somatic mutations and polyreactivity ${ }^{16}$. The field has now been invigorated by new techniques for generating human monoclonal antibodies ${ }^{17,18}$, by the selection of new monoclonal antibodies with particular specificities ${ }^{19-21}$, by new insights into host controls of $\mathrm{BnAb}$ expression ${ }^{16,22,23}$ and by advances in the understanding of the structure of epitopes on the HIV-1 envelope to which BnAbs are directed ${ }^{19,21,24-28}$

It is now clear that $\sim 20 \%$ of patients chronically infected with HIV-1 make BnAbs, although it takes them 3-4 years to do $\mathrm{so}^{29}$. It is now possible to isolate and characterize these antibodies, even though they form a tiny minority of the total antibody response to Env in any patient ${ }^{19,21,25-28,30-32}$. Thus, through the cloning of antigen-specific $B$ cells or by PCR amplification of genes encoding antibodies in plasmablasts obtained from infected patients, it has been possible to characterize hundreds of new monoclonal antibodies that are broadly cross-reactive in their specificities. These studies have identified the CD4-binding site, the membrane-proximal region, a V2V3 conformational (quaternary) epitope and Env glycans as the main target sites for BnAbs ${ }^{19,21,25-28,30-34}$. In many cases, the exact positioning of the antibody on the Env protein is understood, which explains often why these antibodies are neutralizing, whereas others that bind nearby are not ${ }^{19,27}$. Furthermore, a published report of an HIV-1infected person who made both BnAbs to the CD4-binding site and V2V3-conformational $\mathrm{BnAbs}^{35}$ has established proof of the concept that it is possible to induce multiple types of BnAbs in the same person.

What has become very clear from studies of the BnAbs themselves is that they are unusual antibodies in that they are transcribed from genes with considerable somatic mutation, often containing more than 50 somatic mutations relative to the sequence of their nearest germline ancestor $21,27,31,36$. They are often polyreactive with non-HIV antigens ${ }^{31,37}$, and for some specificities, such as the V2V3 conformational epitope, they frequently have extremely long $\mathrm{V}_{\mathrm{H}}$ CDR3 regions ${ }^{13,16}$. Allelic variants of $\mathrm{V}_{\mathrm{H}}$ can be one determinant of germline ancestors of $\mathrm{BnAb}$ recognition of $\mathrm{Env}^{38}$.

When the germline-encoded ancestors of the BnAbs to HIV-1 Env are derived, they often react only with low or undetectable 
affinity with the HIV-1 envelope ${ }^{19,38-41}$. However, it is clear that in the primary B cell response to antigen, the $\mathrm{B}$ cell clones with receptors that bind with the highest affinity outcompete the rest ${ }^{37,42}$. The low affinity of these ancestral antibodies might explain in part why the BnAbs are such a minority population in the mature antibody response to Env.

Immune-tolerance mechanisms are responsible for much of the control of the production of antibodies with the BnAb traits of polyreactivity, long $\mathrm{V}_{\mathrm{H}} \mathrm{CDR} 3$ regions and extensive somatic hypermutations ${ }^{16}$. That last trait might explain why it takes a long time (2-3 years) for such antibodies to appear and why they are more prevalent in patients with high virus loads, in whom there might be a greater antigenic stimulus. It has been proposed that BnAbs may naturally arise only in subjects with relatively relaxed immunetolerance controls ${ }^{35,37}$ and that one way to induce such antibodies by a vaccine is to design artificial immunogens that specifically stimulate ancestral and early-intermediate antibodies of a BnAb clonal lineage ${ }^{39,41,43}$.

Therefore, for vaccine design, the critical question is whether it is possible to 'train' the primary B cell response to favor the development of those specific BnAbs. Can the evolution of antibodies be guided in a way that has not been attempted before? One approach might be to start with a modified immunogen that is based on optimal binding to ancestor antibodies and then to change the immunogen slightly by prime-and-boost strategies ${ }^{43}$. This approach has not been successful before but should be possible, in theory. However, approaches focused solely on the immunogen without consideration of host immunoregulatory controls have not worked so far. Therefore, probing the host-maturation pathways of BnAbs and recreating those pathways with targeted prime-boost immunizations will be a critical requirement for future vaccine design ${ }^{33,43}$.

One radically different approach to this problem is to use vectors to deliver the antibodies. This has worked in monkeys, in which delivery of modified neutralizing antibodies via adenovirus-associated virus protects monkeys from infection with the SIVmac316 strain ${ }^{44}$. In a further development of this delivery approach, long-lived expression of full-length $\mathrm{BnAb}$ has been achieved in humanized mice, which are fully protected against HIV-1 infection ${ }^{36}$.

\section{Vaccines that induce $T$ cell responses}

Alongside the B cell approaches described above, a strategy is needed to make vaccines

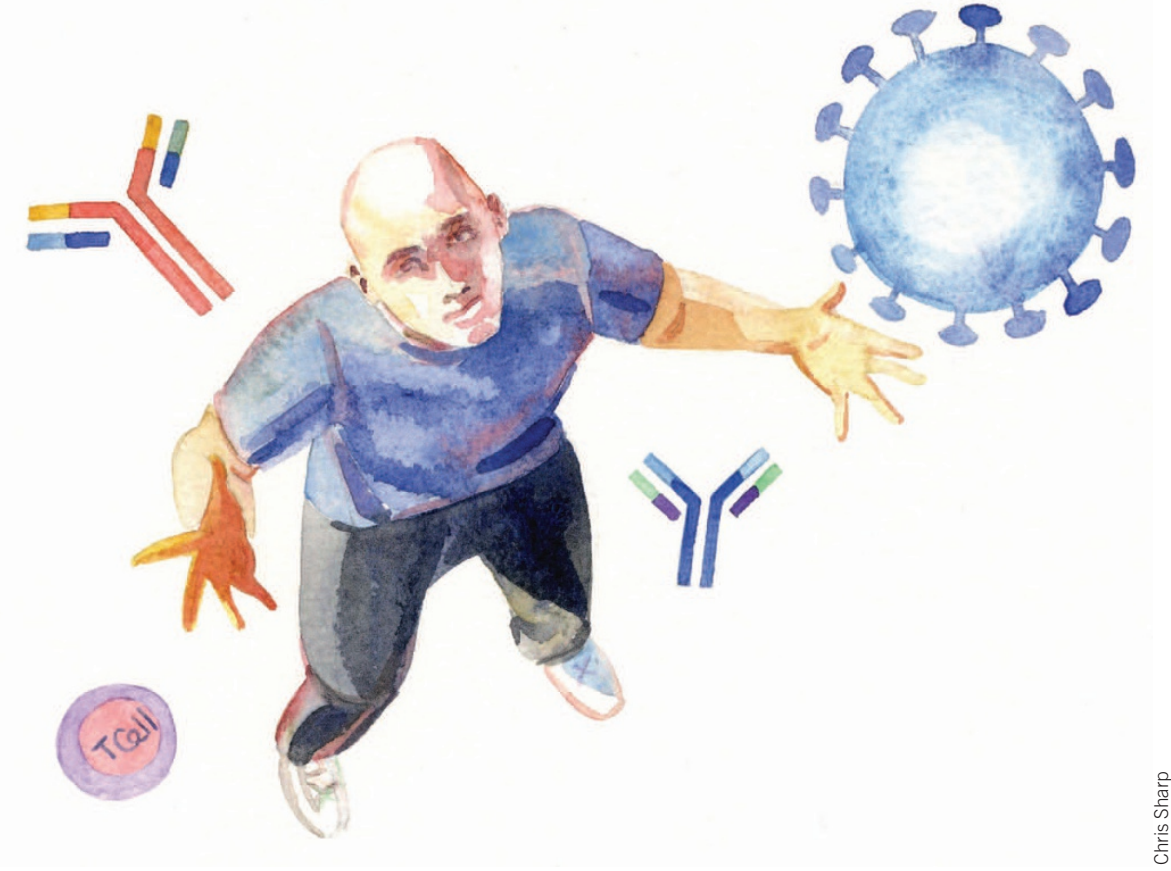

A successful HIV vaccine will probably juggle broadly neutralizing antibodies with strong T cell responses to achieve efficacy.

that stimulate more effective $\mathrm{CD}^{+} \mathrm{T}$ cell and $\mathrm{CD} 4^{+} \mathrm{T}$ cell responses than seen in the STEP trial of the MRK rAd5 vaccine. After a long period during which the SIV-macaque model suggested this was a valid procedure, the results of the phase IIB efficacy (STEP) trial were a real shock ${ }^{3}$. The MRK rAd5 vaccine, which consists of HIV-1 Gag, Pol and Nef expressed in three recombinant adenovirus 5 (rAd5) constructs, stimulated what were thought to be strong $\mathrm{CD}^{+} \mathrm{T}$ cell responses, yet the vaccine failed to offer either protection from the acquisition of infection or good control of the infecting virus ${ }^{3,45}$. The trial was also complicated by the finding that preexisting immunity to the vector, adenovirus 5, enhanced the risk of acquisition of infection with HIV-1, although the effect waned over time ${ }^{46}$. This effect was independent of other risk factors such as lack of circumcision in males $^{46}$

Although the failure to protect was a setback, sieve analysis of breakthrough infections in the STEP trial has demonstrated that vaccine-induced immune pressure can be detected by the identification of virus-escape mutants to vaccine-induced $\mathrm{T}$ cell responses ${ }^{47}$. This has provided some encouragement that the $\mathrm{CD} 8^{+} \mathrm{T}$ cell-stimulating vaccine might still be worth exploring. Recombinant adenovirus vaccines chosen to stimulate much stronger $\mathrm{T}$ cell responses partially protect monkeys against challenge with SIVmac239 and result in very good long-term virus control ${ }^{48}$. The virus used for the challenge is important, as it has become clear that more-natural viruses are much harder to protect against than are the hybrid SHIVs. Subsequently, it was shown that immunization with a recombinant replicating cytomegalovirus vector expressing SIV antigens provided almost complete protection from post-infection SIV viremia in half the macaques subsequently challenged with SIVmac239 (ref. 49). The monkeys were infected, but many had less early viremia, and half seemed to show complete control of SIV viremia. That result has fostered the idea that if sufficiently strong $\mathrm{T}$ cell responses in the right state of activation can be induced, they could be effective. Further support for that proposal has been provided by a study of macaques immunized with adenovirus vectors expressing Env, Gag and Pol, with or without recombinant poxvirus boosts, then repeatedly challenged mucosally with low doses of the neutralization-resistant SIVmac251 strain ${ }^{50}$. Monkeys that received heterologous prime-boost combinations of DNA-MVA, Ad26-Ad35 or Ad26-MVA were partially protected from acquisition of infection, and vaccinated monkeys that did become infected controlled the virus more effectively, especially those primed with Ad26 and boosted with MVA. The protection against acquisition was dependent on expression of Env by the vaccine. Protection from acquisition was associated with Env-binding and tier 1 strain-neutralizing antibodies, and viremic control was most closely associated 
with the magnitude and breadth of $\mathrm{T}$ cell responses to Gag. Because of the results of the RV144 correlates study ${ }^{9}$, this study of nonhuman primates also evaluated antibodies to V2 and found that, as in the RV144 trial, these responses to Env also correlated with infection risk.

Although the results noted above are encouraging, virus variability remains a considerable challenge for the development of vaccines against HIV-1. The vaccine and virus must match sufficiently for $\mathrm{T}$ cells induced by the vaccine to be effective; this probably requires a sequence match of $>90 \%$ between virus and vaccine ${ }^{51}$. Even more critical is rapid eradication of the virus; if it is not eradicated, it may mutate to escape the T cell responses (viral escape), which then become ineffective. There is evidence that this happened in the STEP trial ${ }^{47}$. It is clear that viral escape is driven by $\mathrm{T}$ cell responses and is influenced by the strength of those responses as well as the variability of the epitope (that is, its ability to escape with a minimal fitness cost $)^{52}$. Therefore, several groups have proposed that vaccines be made that focus the $\mathrm{T}$ cell response to the most conserved regions of HIV-1, which would lessen the chance of viral escape because it would impose a serious fitness cost on the virus ${ }^{53-55}$. This can be done by limiting the immunogen to conserved parts of HIV-1 (refs. 53-55) or by broadening the $\mathrm{T}$ cell responses to stimulate responses to those normally subdominant epitopes as well as the commoner variable epitopes. Mosaic vaccines that include common sequence variants have proven successful in inducing $\mathrm{CD} 4^{+} \mathrm{T}$ cell and $\mathrm{CD} 8^{+} \mathrm{T}$ cell responses of much greater breadth and depth in rhesus macaques than do vaccines that contain only consensus or wild-type HIV genes $^{56-58}$. Which of those two approaches is better will be decided by determining whether $\mathrm{T}$ cell responses to conserved epitopes are more effective in the absence of immunodominant responses to more variable regions or not. This can be assessed by comparison of antiviral $\mathrm{CD}^{+} \mathrm{T}$ cell responses in clinical vaccine trials with human subjects. The conserved-epitope approach is already in a phase I clinical trial (Hanke, T. et al., data not shown), and several mosaic HIV-1 genevaccine clinical trials should begin in early 2013 (Haynes, B.F. et al. and Robb, M.L. et al., data not shown).

There is a certain symmetry in the present $\mathrm{B}$ cell and $\mathrm{T}$ cell vaccine approaches. Each approach attempts to circumvent virus variability and to ensure good matching to the circulating viruses and then to ensure that immunity is sustained and not compromised by escape. Each aims to target the most conserved segments of the virus. In each case, the responses are normally subdominant and thus must be artificially enhanced. Each immune response must be sufficiently robust so that after vaccination, the same response is recalled and is not overwhelmed by new primary responses when the virus strikes. There is every reason to believe that these approaches could work, but considerable ingenuity will be needed to guide both B cell and $\mathrm{T}$ cell responses through the desired but unfavored maturation pathways.

What else is possible? The results of the RV144 trial remain intriguing and should lead to the consideration of other approaches. A major hypothesis is that some form of antibody-dependent antiviral effect mediated by cells expressing the receptor for the $\mathrm{Fc}$ fragment was involved in the protection against acquisition noted in the RV144 trial. However, although such non-neutralizing antibody responses are induced by all Env vaccines, their protective qualities are hard to confirm because of their lack of direct antiviral activity in vitro and only indirect evidence in vivo ${ }^{59}$. New models of rhesus macaques challenged with a low dose of SIVHIV hybrid viruses that encode HIV-1 Env that uses the receptor CCR5 are needed to determine the full extent of protection mediated by non-neutralizing antibodies to HIV-1 Env.

Finally, a broader approach to innate immunity might be worth considering. There are both inhibitory and activating receptors on natural killer cells that respond to complexes of peptide and major histocompatibility complex. Could these be harnessed with a vaccine? There is some evidence that innate memory exists and that it can protect against viral infection ${ }^{60,61}$. However, much more must be known about the specificity of the natural killer cell receptors, particularly those used by the activating killer immunoglobulin-like receptors, before such vaccines can be designed. Also the possibility of modulating natural killer $\mathrm{T}$ cells ${ }^{62}$ and regulatory $\mathrm{T}$ cells ${ }^{63,64}$, diminishing loss of the $\mathrm{T}_{\mathrm{H}} 17$ subset of helper $\mathrm{T}$ cells in the gut ${ }^{65}$ and decreasing $\mathrm{T}$ cell activation ${ }^{66}$ during acute infection with HIV-1 could be worth exploring further.

Overall, there is now considerable new optimism in the HIV-1 vaccine-development field because of the remarkable progress in research over the past 3 years. Although a timetable for the creation of a usable preventive vaccine is still some way off, the coming years should see many of these new discoveries and insights translated into new vaccine candidates and approaches. If enhanced efficacy can be seen in the next post-RV144 efficacy trials of vaccines and/or if progress can be made in learning to induce large numbers of mucosal BnAbs, coupled with continued progress in the development of $\mathrm{T}$ cell-inducing vaccines that overcome HIV-1 diversity, then the timelines for final vaccine development will become clear. A vaccine is not around the corner, but it is definitely closer than it was a few years ago.

\section{ACKNOWLEDGMENTS}

We thank A. Duerr, N. Michael, G. Tomaras, J. Kim, B. Hahn, G. Kelsoe, T. Hanke and N. Goonetilleke for discussions. Supported by the Division of AIDS of the National Institute of Allergy and Infectious Diseases of the US National Institutes of Health (AI067854 for the Center for HIV/AIDS Vaccine Immunology), the Collaboration for AIDS Vaccine Discovery Vaccine Development Center of the Bill and Melinda Gates Foundation (B.F.H.), the UK Medical Research Council and the UK National Institute for Health Research.

\section{COMPETING FINANCIAL INTERESTS}

The authors declare competing financial interests: details accompany the full-text HTML version of the paper at http://www.nature.com/natureimmunology/.

\section{Corrected after print 20 April 2012.}

1. Gilbert, P.B. et al. J. Infect. Dis. 191, 666-677 (2005).

2. Pitisuttithum, P. et al. J. Infect. Dis. 194, 1661-1671 (2006).

3. Buchbinder, S.P. et al. Lancet 372, 1881-1893 (2008).

4. Rerks-Ngarm, S. et al. N. Engl. J. Med. 361, 22092220 (2009).

5. Kim, J.H., Rerks-Ngarm, S., Excler, J.L. \& Michael, N.L. Curr. Opin. HIV AIDS 5, 428-434 (2010).

6. Cohen, J. Science 326, 652-653 (2009).

7. Gilbert, P.B. et al. J. Infect. Dis. 203, 969-975 (2011).

8. Montefiori, D. et al. J. Infect. Dis. (in the press) (2012).

9. Haynes, B.F. et al. N. Engl. J. Med. 366, 1275-1286 (2012).

10. Price, M.A. et al. J. Acquir. Immune Defic. Syndr. $\mathbf{5 9}$ 185-193 (2012)

11. Keele, B.F. et al. Proc. Natl. Acad. Sci. USA 105 $7552-7557$ (2008)

12. Forthal, D.N., Gilbert, P.B., Landucci, G. \& Phan, T. J. Immunol. 178, 6596-6603 (2007).

13. Dinges, W.L. et al. J. Virol. 84, 4461-4468 (2010).

14. Burton, D.R. \& Weiss, R.A. Science 329, 770-773 (2010)

15. McMichael, A.J., Borrow, P., Tomaras, G.D., Goonetilleke, N. \& Haynes, B.F. Nat. Rev. Immunol. 10, 11-23 (2010)

16. Verkoczy, L., Kelsoe, G., Moody, M.A. \& Haynes, B.F. Curr. Opin. Immunol. 23, 383-390 (2011).

17. Wardemann, H. et al. Science 301, 1374-1377 (2003)

18. Wrammert, J. et al. Nature 453, 667-671 (2008).

19. Scheid, J.F. et al. Science 333, 1633-1637 (2011).

20. Morris, L. et al. PLoS ONE 6, e23532 (2011).

21. Wu, X. et al. Science 329, 856-861 (2010).

22. Verkoczy, L. et al. J. Immunol. 187, 3785-3797 (2011)

23. Verkoczy, L. et al. Proc. Natl. Acad. Sci. USA 107 181-186 (2010)

24. Pejchal, R. et al. Science 334, 1097-1103 (2011).

25. Chen, L. et al. Science 326, 1123-1127 (2009).

26. McLellan, J.S. et al. Nature 480, 336-343 (2011).

27. Wu, X. et al. Science 333, 1593-1602 (2011).

28. Zhou, T. et al. Science 329, 811-817 (2010). 
29. Gray, E.S. et al. J. Virol. 85, 4828-4840 (2011). 30. Diskin, R. et al. Science 334, 1289-1293 (2011). 31. Mouquet, H. et al. Nature 467, 591-595 (2010). 32. Walker, L.M. et al. Nature 477, 466-470 (2011).

33. Bonsignori, M. et al. J. Virol. 85, 9998-10009 (2011).

34. Corti, D. et al. PLoS ONE 5, e8805 (2010).

35. Bonsignori, M. et al. J. Virol. published online doi:10.1128/JVI.07163-11 (1 February 2012).

36. Balazs, A.B. et al. Nature 481, 81-84 (2011).

37. Haynes, B.F. et al. Science 308, 1906-1908 (2005).

38. Alam, S.M. et al. J. Virol. 85, 11725-11731 (2011).

39. Xiao, X. et al. Biochem. Biophys. Res. Commun. 390, 404-409 (2009).

40. Liao, H.X. et al. J. Exp. Med. 208, 2237-2249 (2011).

41. Ma, B.J. et al. PLoS Pathog. 7, e1002200 (2011).
42. Shih, T.A., Meffre, E. Roederer, M. \& Nussenzweig M.C. Nat. Immunol. 3, 570-575 (2002).

43. Haynes, B. et al. Nat. Biotechnol. (in the press) (2012).

44. Johnson, P.R. et al. Nat. Med. 15, 901-906 (2009). 45. McElrath, M.J. et al. Lancet 372, 1894-1905 (2008). 46. Duerr, A. et al. J. Infect. Dis. (in the press) (2012).

47. Rolland, M. et al. Nat. Med. 17, 366-371 (2011).

48. Liu, J. et al. Nature 457, 87-91 (2009).

49. Hansen, S.G. et al. Nature 473, 523-527 (2011).

50. Barouch, D.H. et al. Nature 482, 89-93 (2012).

51. Lee, J.K. et al. J. Exp. Med. 200, 1455-1466 (2004).

52. Ferrari, G. et al. PLoS Pathog. 7, e1001273 (2011).

53. Létourneau, S. et al. PLoS ONE 2, e984 (2007).

54. Rolland, M., Nickle, D.C. \& Mullins, J.I. PLoS Pathog. 3, e157 (2007).

55. Mothe, B. et al. J. Transl. Med. 9, 208 (2011).
56. Korber, B. T., Letvin, N.L. \& Haynes, B.F. J. Virol 83 8300-8314 (2009)

57. Santra, S. et al. Nat. Med. 16, 324-328 (2010). 58. Barouch, D.H. et al. Nat. Med. 16, 319-323 (2010). 59. Burton, D.R. et al. Proc. Natl. Acad. Sci. USA 108 11181-11186 (2011).

60. Sun, J.C., Beilke, J.N. \& Lanier, L.L. Nature 457 557-561 (2009).

61. Gillard, G.O. et al. PLoS Pathog. 7, e1002141 (2011)

62. Mureithi, M.W. et al. AIDS Res. Hum. Retroviruses 27, 501-509 (2011).

63. Favre, D., et al. Sci. Transl. Med. 2, 32 ra36 (2010) 64. Elahi, S. et al. Nat. Med. 17, 989-995 (2011).

65. Brenchley, J.M. et al. Blood 112, 2826-2835 (2008).

66. Estes, J.D. et al. PLoS Pathog. 6, e1001052 (2010). 


\section{Erratum: Lessons learned from HIV-1 vaccine trials: new priorities and directions}

Andrew J McMichael \& Barton F Haynes

Nat. Immunol. 13, 423-427 (2012); published online 18 April 2012; corrected after print 20 April 2012

In the version of this article initially published, the second sentence of the abstract and reference 9 were incorrect. That second sentence should read "Correlates of risk of infection for the RV144 trial have been found." Reference 9 should read "Haynes, B.F. et al. N. Engl. J. Med. 366, 1275-1286 (2012)." The errors have been corrected in the HTML and PDF versions of the article. 